\title{
Cirugía durante la pandemia del SARS-COV-2 / COVID-19: el efecto de la generación de aerosoles de partículas en escenarios quirúrgicos
}

\author{
Surgery during the SARS-COV-2 / COVID-19 pandemic: the effect of \\ particle aerosol generation on surgical scenarios
}

Luis Felipe Cabrera ${ }^{1}$, Mauricio Pedraza Ciro ${ }^{2}$, Lilian Torregrosa ${ }^{3}$, Edgar Figueredo ${ }^{4}$

1 Médico, Especialista en Cirugía General, Departamento de Cirugía, Fundación Santa Fe de Bogotá. Profesor de cirugía, Universidad El Bosque y Universidad de los Andes. Bogotá, Colombia. Miembro Comité de Comunicaciones Asociación Colombiana de Cirugía Médico, Residente de Cirugía General, Universidad El Bosque. Bogotá, Colombia.

3 Médica, Especialista en Cirugía General y Cirugía de Mama y tejidos blandos. Directora, Departamento de Cirugía, Pontificia Universidad Javeriana - Hospital Universitario San Ignacio. Bogotá, Colombia. Presidente Asociación Colombiana de Cirugía.

4 Médico, Especialista en Cirugía General, Profesor Asociado de Cirugía General, Cuidado Intensivo Quirúrgico y Simulación en medicina. University of Washington School of Medicine. Seattle, Estados Unidos.

\section{Resumen}

Hasta el momento no se ha publicado información conclusiva que respalde la teoría de que los virus respiratorios se transmitan a través del humo quirúrgico o el neumoperitoneo. Por lo tanto, las alertas sobre los riesgos de la laparoscopia emitidas durante las primeras semanas de la pandemia deben ser analizadas con precaución y a la luz de la evidencia cambiante sobre el tema. Tanto la cirugía abierta como la laparoscópica tienen el potencial de generar aerosoles de partículas y por lo tanto, en ambos escenarios es fundamental la protección de todo el equipo de trabajo dentro del quirófano. En todos los procedimientos durante la época de pandemia, se deben buscar las estrategias más efectivas para controlar las potenciales fuentes de trasmisión y minimizar la exposición del personal en los momentos de mayor riesgo, relacionados con el manejo de la vía aérea y las cavidades del paciente. La siguiente es una revisión narrativa de literatura sobre las cirugías durante la pandemia del SARS-CoV-2 / COVID-I9 y el efecto de los aerosoles durante estos procedimientos, con el fin de integrar y dar a conocer las principales medidas que se han propuesto a nivel global, para manejar este nuevo grupo de pacientes.

Palabras clave: COVID-I9; virus del SARS Cov 2; pandemia; cirugía; laparoscopía; aerosoles.

Fecha de recibido: 13/04/2020 - Fecha de aceptación: 16/04/2020

Autor de correspondencia: Luis Felipe Cabrera-Vargas. Dirección: Calle 157 \# 13 B - 20 Casa 10 Conjunto Almeria. Bogotá DC, Colombia. Celular: $311-4342746$

Correo electrónico: luis.felipe.cabrera@hotmail.com

Citar como: Cabrera LF, Pedraza-Ciro M, Torregrosa L, Figueredo E. Cirugía durante la pandemia del sars-cov-2/COVID-19: el efecto de la generación de aerosoles de partículas en escenarios quirúrgicos. Rev Rev Colomb Cir. 2020;35:190-9/Especial COVID-19. https://doi.org/10.30944/20117582.625

Este es un artículo de acceso abierto bajo una Licencia Creative Commons - BY-NC-ND https://creativecommons.org/licenses/by-ncnd/4.0/deed.es 


\begin{abstract}
To date no conclusive information has been published to support the theory that respiratory viruses are transmitted through surgical smoke or pneumoperitoneum. Therefore, alerts about the risks of laparoscopy issued during the first weeks of the pandemic should be viewed with caution and in light of changing evidence on the subject. Both open and laparoscopic surgery have the potential to generate aerosols of particles and therefore, in both scenarios, the protection of all the surgical team within the operating room is essential. In all procedures during the pandemic season, the most effective strategies should be sought to control potential sources of transmission and minimize staff exposure at times of greatest risk related to the management of the patient's airway and cavities. The following is a narrative review of the literature on surgeries during the SARS-CoV-2 / COVID-I9 pandemic and the effect of aerosols during these procedures, in order to integrate and publicize the main measures that have been proposed globally, to manage this new group of patients.
\end{abstract}

Keywords: COVID-I9; SARS Cov 2 virus; pandemic; surgery; laparoscopy; aerosols.

\section{Introducción}

Los aerosoles son partículas menores de $5 \mu \mathrm{m}$ de diámetro que se producen cuando una corriente de aire se mueve a través de una superficie con película de líquido; su tamaño es inversamente proporcional a la velocidad del aire y pueden permanecer en el ambiente por largos periodos, así como recorrer distancias mayores a un metro ${ }^{\mathrm{I}, 2}$.

Un procedimiento generador de aerosol (PGA) es definido como aquel procedimiento médico que puede inducir la producción de aerosoles de cualquier tamaño, incluyendo los núcleos de gotas ${ }^{\perp}$. William F. Wells describió en 1930 la transmisión de enfermedades respiratorias como la tuberculosis a través de las emisiones de microgotas respiratorias, divididas en microgotas "grandes" y "pequeñas" 2

Es importante definir y categorizar los procedimientos que generan aerosoles ya que éstos pueden estar relacionados con la propagación de la enfermedad, especialmente en los profesionales de la salud, en quienes las enfermedades ocasionadas por partículas virales pueden ser transmitidas, además de las rutas de contacto, por aerotransportación a través de las gotas respiratorias. Si bien en un análisis de más de 75.000 casos en China no se pudo documentar el contagio directo por aerosoles, la transmisión a través de este mecanismo es considerada plausible en circunstancias específicas y en ambientes donde se realizan PGA 3 .
La siguiente es una revisión narrativa de literatura sobre las cirugías durante la pandemia del SARS-CoV-2 / COVID-I9 y el efecto de los aerosoles durante estos procedimientos (figura I), con el fin de integrar y dar a conocer las principales medidas que se han propuesto a nivel global, para manejar este nuevo grupo de pacientes.

\section{Procedimientos generadores de aerosol (PGA)}

La transmisión por aerosoles se produce por la diseminación de núcleos de microgotas en el aire, o de pequeñas partículas de tamaño respirable, que contienen agentes patógenos, los cuales permanecen infecciosos a lo largo del tiempo y pueden alcanzar distancias iguales o superiores a I metro (ej, esporas de Aspergillus spp). Algunos de los microorganismos transportados de esta manera pueden dispersarse incluso a grandes

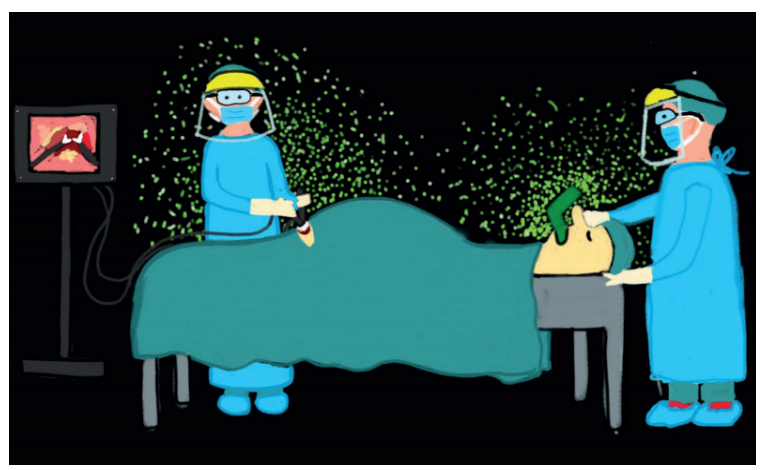

Figura 1. Focos de mayor generación de aerosoles durante la cirugía. 
distancias a través de corrientes de aire y pueden ser inhalados por personas susceptibles que no hayan tenido contacto cara a cara con el individuo infeccioso ${ }^{4-6}$.

En contraste con la interpretación estricta de una ruta de transmisión aérea, se ha demostrado la transmisión a corta distancia por medio de aerosoles de pequeñas partículas generados en circunstancias específicas (por ejemplo, durante la intubación endotraqueal), a personas que se encuentran a menos de 2 metros del paciente. Adicionalmente, las partículas en aerosol $(<\mathrm{IOO} \mu \mathrm{m})$, pueden también permanecer en suspensión en el aire, cuando las velocidades de la corriente de aire de la habitación superan la velocidad de asentamiento terminal de las partículas ${ }^{7,8}$.

Una reciente revisión no sistemática presenta los siguientes como procedimientos generadores de aerosoles: administración de medicamentos en nebulización o aerosol, inducción diagnóstica del esputo, broncoscopia; succión de la vía aérea, intubación endotraqueal, ventilación de presión positiva (ej. CPAP), ventilación oscilatoria de alta frecuencia, reemplazo de tubo endotraqueal o tubo de traqueostomía y actividades relacionadas con la desconexión del sistema ventilatorio (incluyendo las desconexiones accidentales) ${ }^{9}$.

La Organización Mundial de la Salud (OMS) asevera que los procedimientos anteriormente mencionados podrían ser generadores de aerosoles ya que tienen la capacidad de transmitir patógenos ${ }^{\mathrm{Io}}$, sin embargo, en procedimientos como administración de medicamentos por nebulización, no se encontró suficiente evidencia que lo respaldara.

En una revisión sistemática realizada por la Agencia canadiense de medicamentos y tecnologías de la salud en el 20II, se identificaron diez estudios pertinentes no aleatorizados para evaluar el riesgo de transmisión de infección respiratoria aguda en PGA. De los anteriores, 5 estudios de casos y controles y 5 estudios de cohortes retrospectivas evaluaron la transmisión del síndrome respiratorio agudo severo (SARS) a los trabajadores de la salud durante la atención de pacientes en hospitales o unidades de cuidados intensivos durante los brotes de SARS de 2002-2003 ${ }^{\text {7, II }}$.

Los procedimientos que mostraron un aumento estadísticamente significativo del riesgo de transmisión del SARS a los trabajadores de la salud o que fueron un factor de riesgo estadísticamente significativo de infección por SARS en este grupo, incluyen la intubación endotraqueal (cuatro estudios de cohorte, odds-ratio [OR] combinado 6,6 con $\mathrm{IC}_{95 \%}$ : 2,3-I8,9 y cuatro estudios de casos y controles, OR combinado de 6,6 con $\mathrm{IC}_{95 \%}$ : 4,I-IO,6); ventilación no invasiva (dos estudios de cohorte, OR combinado 3,I con $\mathrm{IC}_{95 \%}$ : I,4-6,8); traqueostomía (un estudio de casos y controles, OR 4,2 con $\mathrm{IC}_{95 \%}:$ I,5-II,5); y ventilación manual antes de la intubación (un estudio de cohorte, OR 2,8 con $\left.\mathrm{IC}_{95 \%}: \mathrm{I}, 3-6,4\right)$. El riesgo de transmisión no tuvo una asociación estadísticamente significativa con la succión antes de la intubación (dos estudios de cohorte, OR combinado 3,5 con $\mathrm{IC}_{95 \%}$ : 0,5-24,6); la succión después de la intubación (dos estudios de cohorte, OR combinado I,3 con $\mathrm{IC}_{95 \%}$ : 0,5-3,4); la ventilación manual después de la intubación (un estudio de cohorte, OR I,3 con $\mathrm{IC}_{95 \%}$ : 0,5-3,2); broncoscopia (dos estudios de cohorte, OR combinado I,9 con $\mathrm{IC}_{95 \%}$ : O,2-I4,2); tratamiento con nebulizador (dos estudios de cohorte, OR combinado 3,7 con $\mathrm{IC}_{95 \%}$ : 0,7-I9,5); manipulación de la máscara de oxígeno (dos estudios de cohorte, OR combinado 4.6 con $\mathrm{IC}_{95 \%}$ : 0,6-32,5); manipulación de la máscara de ventilación mecánica no invasiva bi level (BiPAP) (un estudio de cohorte, OR 4,2 con $\mathrm{IC}_{95 \%}$ : O,6427,4); desfibrilación (dos estudios de cohorte, OR combinado 2,5 con $\mathrm{IC}_{95 \%}$ : O,I-43,9); compresiones torácicas (dos estudios de cohorte, OR combinado I,4 con $\mathrm{IC}_{95 \%}$ : 0,2-II,2); la inserción de una sonda nasogástrica (dos estudios de cohorte, OR combinado I,2 con $\mathrm{IC}_{95 \%}$ : 0,4-4,0); y la obtención de una muestra de esputo (un estudio de cohorte, OR 2,7 con $\mathrm{IC}_{95 \%}$ : $\left.0,9-8,2\right)^{7, \mathrm{II}}$.

Además, la ventilación oscilatoria de alta frecuencia (un estudio de cohorte, OR 0,7 con $\mathrm{IC}_{95 \%}$ : o,I-5,5); el oxígeno de alto flujo (un estudio de cohorte, OR 0,4 con $\mathrm{IC}_{95 \%}$ : O,I-I,7); la aspiración endotraqueal (un estudio de cohorte, OR I,O con 
$\mathrm{IC}_{95 \%}$ : 0,2-5,2); la succión de fluidos corporales (un estudio de casos y controles, OR I,O con $\mathrm{IC}_{95 \%}$ : o,4-2,8); la administración de oxígeno (un estudio de casos y controles, OR I,I con $\mathrm{IC}_{95 \%}$ : 0,4-2,8); la toma de muestras de esputo (un estudio de cohortes, OR 2,7 con $\mathrm{IC}_{95 \%}$ : 0,9-8,2); la fisioterapia torácica (dos estudios de cohorte, OR combinado 0,8 con $\mathrm{IC}_{95 \%}$ : 0,2-3,2); y la ventilación mecánica (un estudio de cohorte, OR o,9 con $\mathrm{IC}_{95 \%}$ : 0,42,0), no mostraron diferencias estadísticamente significativas en el riesgo de transmisión, pero todos los estudios se calificaron como de muy baja calidad según la evaluación de evidencia GRADE ${ }^{7, \mathrm{II}}$.

Zemouri y colaboradores, en una revisión sistemática sobre el alcance de los bio-aerosoles en la salud y el entorno dental, analizaron 17 estudios sobre la composición microbiana, encontrando que la carga bacteriana media en los bio-aerosoles oscilaba entre Log $\mathrm{I} \pm 3.9 \mathrm{CFU} / \mathrm{m}^{3}$. Además, seis estudios analizaron la contaminación del bio-aerosol antes y después del tratamiento. La carga bacteriana o fúngica varió de $\log -0.7 \pm 2.4$ $\mathrm{UFC} / \mathrm{m} 3$ en la línea de base, a Log $\mathrm{I} \pm 3, \mathrm{I}$ UFC/ $\mathrm{m}^{3}$ después del tratamiento. Sólo un estudio informó sobre la relación entre la distancia de la fuente generadora de bio-aerosoles y la carga bacteriana. Ellos encontraron una mayor carga bacteriana en los bio-aerosoles a I, 5 metros de la cavidad oral del paciente que en los bio-aerosoles a menos de I metro del paciente. Un estudio se realizó para Burkholderia cepacia y otro para $M$. tuberculosis, y ninguno pudo recuperar estos microorganismos después del tratamiento regular del paciente ${ }^{8}$.

En la literatura revisada es evidente la falta de precisión en la determinación de PGA, así como la falta de evidencia en relación a la transmisión confirmada de esta enfermedad a través de algunos de esos procedimientos 9,II.

Existen además importantes vacíos en lo que respecta a la epidemiología de la transmisión de las infecciones respiratorias agudas durante los PGA, desde los pacientes hacia los trabajadores de la salud, y en particular en lo que respecta a los patógenos distintos del SARS-CoV-2
/ COVID-I9. Es escasa la información sobre los requisitos mínimos de ventilación que podrían reducir en forma efectiva la transmisión de patógenos durante estos procedimientos, y aunque existen múltiples recomendaciones al respecto, la calidad de la evidencia que las sustenta no es la mejor.

Actualmente, se considera que la mejor práctica para mitigar la posible transmisión infecciosa durante los procedimientos abiertos, laparoscópicos y endoscópicos, es utilizar un enfoque combinado que idealmente incluya la filtración y ventilación adecuadas de la sala, el uso de los elementos de protección personal (EPP) apropiados y los dispositivos de evacuación de humo con un sistema de succión y filtración ${ }^{\text {. }}$

Los momentos de cambio de los elementos de protección personal (colocación y retiro) conllevan un mayor riesgo de exposición a los aerosoles, por lo que se recomienda el uso de un "sistema de amigos", ubicándose en parejas para que otra persona de la misma área pueda hacer la supervisión y retroalimentación de las acciones tomadas por su compañero de equipo durante estos momentos ${ }^{6, \mathrm{II}, 12}$.

De acuerdo con la información planteada en las diferentes guías, proponemos la siguiente clasificación de los procedimientos, que toma como parámetro la evidencia a la fecha sobre la capacidad que tiene cada uno de ellos para generar aerosoles y la potencial transmisión de partículas. Los procedimientos e intervenciones se clasificarían en dos grandes grupos denominados "generadores" y "potencialmente generadores" de aerosoles. En la tabla I se resumen los procedimientos que se realizan dentro del quirófano o fuera de el.

\section{Procedimientos quirúrgicos}

Hasta el día de hoy (I6 de abril de 2020) no se ha publicado evidencia científica conclusiva que demuestre un mayor riesgo de transmisión de SARS-CoV-2 / COVID-I9 con la cirugía laparoscópica y permita concluir que se debe preferir el abordaje abierto ${ }^{13}$. Sin embargo, el alto volumen 
Tabla 1. Clasificación de los procedimientos, basada en la evidencia que soporta la capacidad de generación de aerosoles y la potencial transmisión de partículas en cada uno de ellos.

\begin{tabular}{|c|c|}
\hline \multicolumn{2}{|c|}{ PROCEDIMIENTOS GENERADORES DE AEROSOLES } \\
\hline \multicolumn{2}{|c|}{ DENTRO DEL QUIRÓFANO } \\
\hline $\begin{array}{l}\text { Generadores de aerosoles } \\
\text { (con posible transmisión) }\end{array}$ & Potencialmente generadores de aerosoles \\
\hline Intubación endotraqueal y extubación & Succión antes y después de la intubación \\
\hline Ventilación manual antes de la intubación & Ventilación manual después de la intubación \\
\hline Toracostomía a drenaje cerrado & Inserción de una sonda nasogástrica \\
\hline \multirow[t]{2}{*}{ Traqueostomía abierta o percutánea } & Actividades en las que se desconecte el sistema ventilatorio \\
\hline & $\begin{array}{l}\text { Todas las cirugías, tanto laparoscópicas como abiertas, en es- } \\
\text { pecial si requieren el uso de electrocirugía (lo cual incluye el uso } \\
\text { del electrobisturí) }(1,4,11)\end{array}$ \\
\hline
\end{tabular}

\section{FUERA DEL QUIRÓFANO}

\section{Generadores de aerosoles (con posible transmisión)}

Intubación endotraqueal o extubación, succión y procedimientos relacionados.

Ventilación no invasiva, incluyendo CPAP, BiPAP, ASV

Ventilación Oscilatoria de Alta Frecuencia (HFOV)

Cánula Nasal de Alto Flujo (HFNO)

Ventilación manual antes de la intubación

Broncoscopia y procedimientos relacionados (broncoscopia rígida, fibrobroncoscopia flexible, cepillado y lavado broncoalveolar, biopsia transbronquial, etc)

Traqueostomía en UCl o en sala de reanimación (colocación, Ecocardiograma transesofágico retiro y cambio de la cánula)

Cricotiroidotomía

Toracostomía a drenaje cerrado de pacientes en UCI o reanimación

Intervenciones en el servicio de urgencias con paciente de trauma

Toracotomía de resucitación

Toracostomía de drenaje cerrado

Sutura de herida penetrante soplante (tórax o cuello)

Procedimientos dentales con uso de dispositivos de alta velocidad

Procedimientos con uso de succión fenestrada o motor/ fresa a altas revoluciones: tracto aerodigestivo superior (oído, cavidad nasal, cavidad oral, faringe y laringe)

Necropsia

CPAP: Presión positiva continua en la vía aérea; BiPAP: Presión positiva doble en la vía aérea; ASV: Servo ventilación adaptativa; UCI: Unidad de Cuidado Intensivo.

\section{Potencialmente generadores de aerosoles}

Tratamiento con nebulizador

Obtención de una muestra de esputo y esputo inducido

Compresiones torácicas

Endoscopia gastrointestinal superior e inferior

Procedimientos endoscópicos y no endoscópicos de la vía aerodigestiva

Extracción de cuerpo extraño en tracto aerodigestivo superior, drenaje de absceso oral

Inserción de una sonda nasogástrica

Actividades en las que se desconecta el sistema ventilatorio en $\mathrm{UCl}$ o reanimación

Trabajo de parto y atención del parto 
de gas potencialmente contaminado que permanece en la cavidad durante la cirugía laparoscópica, al momento de su liberación puede generar una mayor exposición del personal quirúrgico, el cual se encuentra en íntimo contacto con el paciente en la mesa de cirugía y dentro del quirófano ${ }^{14}$. Lo anterior fue reportado en un estudio, en donde tras el uso de electricidad o ultrasonido en laparoscopia por Io minutos, la concentración de las partículas fue significativamente mayor que en cirugía abierta ${ }^{15}$.

La Society of American Gastrointestinal and Endoscopic Surgeon (SAGES), el American College of Surgeons (ACS) y el Royal College of Surgeons of England (RCS) en sus recomendaciones de finales de marzo y comienzos de abril de 2020 sugieren que al escoger la vía de abordaje para cirugía se tengan en cuenta, además de la posibilidad de generación de aerosoles, los beneficios de cada una de las vías de acceso, como son el tiempo de recuperación y la estancia hospitalaria, ya que esto podría limitar riesgos de contagio para el paciente y el equipo médico, así como beneficiar la capacidad hospitalaria en tiempos de crisis ${ }^{16}$. En cuanto a cirugía ginecológica, se considera prudente realizar procedimientos vaginales y vía abierta bajo anestesia regional para evitar eventos de intubación y extubación que generan aerosoles. Sin embargo, la ruta quirúrgica depende de las comorbilidades de la paciente.

También se considera realizar disección y control vascular usando técnicas no electroquirúrgicas, mientras sea posible, y utilizar extractores de humo junto con filtros de alta eficiencia ${ }^{17}, 18$. El uso de un sistema de evacuación / filtración de humo permite la liberación controlada y la filtración de gases y humo quirúrgico. Idealmente, esto se logra con el uso de un filtro de aire de partículas ultrabajo (ULPA), clasificado para filtrar partículas de o.I micras de diámetro, el cual puede eliminar hasta el $99.999 \%$ de las partículas en el aire, con un tamaño mínimo de penetración de partículas de 0.05 micras. Por el contrario, los filtros de aire de alta eficiencia (HEPA) solo filtran partículas de más de 0.3 micras de diámetro ${ }^{18}$.

\section{Cirugía mínimamente invasiva}

No se encuentran reportes en la literatura sobre el riesgo específico de trasmisión del virus SARSCoV-2 / COVID-I9 en cirugía de mínima invasión, por lo que, para emitir recomendaciones específicas sobre las medidas de control del riesgo de trasmisión, se debe tener en cuenta lo que conocemos hasta ahora con respecto al SARSCoV-2 / COVID-19 9,12,13,19.

Debemos establecer que, basados en la mejor evidencia disponible, el SARS-CoV-2 COVID-I9, es un patógeno respiratorio. Tanto el ARN como el virus infeccioso se detectan principalmente en muestras del tracto respiratorio. Los viriones COVID-I9 tienen un tamaño de aproximadamente 0,I25 micras y se transmiten más comúnmente como gotas de agua respiratoria más grandes (> 20 micras). El virus también puede ser aerosolizado y transmitido en pequeñas gotas $(<\mathrm{IO}$ micras) en suspensión de gas. El tamaño de las partículas tiene implicaciones para el tiempo de suspensión y los requisitos de filtración. El ARN del SARS-CoV-2 / COVID-I9 también se ha detectado en muestras de sangre y heces, pero se desconoce si el virus infeccioso está presente en estas muestras extrapulmonares $9,12,13,19$. Hasta la fecha, ningún estudio ha identificado el SARSCoV-2 en humo quirúrgico, e incluso si se encuentra, no se sabe si estas partículas virales son infecciosas. Además, aunque se puede detectar ARN viral en la sangre, no se ha documentado ninguna transmisión de SARS-CoV-2 / COVID-I9 a través de esta ruta ${ }^{13,20-22}$.

En retrospectiva, hemos podido aprender de otras epidemias virales, que el riesgo de transmisión de enfermedades virales con la laparoscopía es prácticamente nulo. Aunque el SARS-CoV-2 / COVID-I9 es una enfermedad nueva, la evidencia de virus respiratorios similares, como la gripe y otros coronavirus (Síndrome Respiratorio Agudo Severo - SARS y Síndrome Respiratorio del Medio Oriente - MERS-CoV), no ha demostrado transmisión de la enfermedad a través del neumoperitoneo ni del humo quirúrgico ${ }^{9,13,21}$.

Por otro lado, el ADN viral de los patógenos transmitidos por la sangre, como la hepatitis B y 
el virus de la inmunodeficiencia humana (VIH), se ha detectado en el humo quirúrgico después del uso de energía durante la cirugía (por ejemplo, electrocirugía), pero no hay evidencia que indique que el uso de electrocirugía durante la laparoscopia aumente el riesgo de transmisión de la enfermedad a través del humo quirúrgico o el neumoperitoneo ${ }^{5,6}$. Además, la cirugía en pacientes con $\mathrm{VIH}$, hepatitis $\mathrm{B}$ y $\mathrm{C}$ ha estado en curso durante décadas, sin documentar un mayor riesgo de transmisión para los cirujanos, anestesiólogos o el resto del personal en el quirófano por el uso de neumoperitoneo. Por lo cual las técnicas laparoscópicas que minimizan significativamente la exposición de los cirujanos a los patógenos transmitidos por la sangre son preferidas a la laparotomía ${ }^{12,19,20-22}$.

Durante la cirugía gastrointestinal el uso del bisturí y la apertura del tracto digestivo pueden generar aerosoles. La laparoscopia supone la interposición de una barrera física entre el cirujano y la posible fuente de contagio, evitándose la exposición directa del cirujano a los aerosoles potencialmente contaminados con el virus y la infección cruzada. Si bien los sistemas de evacuación y filtración de humo se pueden usar tanto en cirugía abierta como laparoscópica, la laparoscopia ofrece la ventaja única de poder contener casi por completo el neumoperitoneo y el humo quirúrgico en la cavidad abdominal. Junto con los puertos laparoscópicos herméticos, se puede utilizar un sistema de evacuación / filtración para minimizar la liberación de virus potenciales en el aire de la sala de cirugía, mientras se evacua simultáneamente el humo quirúrgico de forma activa o pasiva. Lo cual contrasta con la evacuación de humo quirúrgico durante una cirugía abierta o una laparotomía, donde la contención del humo quirúrgico es desafiante o casi imposible. El evacuador de humo quirúrgico en cirugía abierta debe estar idealmente a un máximo de 2 $\mathrm{cm}$ de la fuente, con una pérdida de captura del humo quirúrgico hasta del $50 \%$ por cada I $\mathrm{cm}$ de distancia de la fuente que lo produce ${ }^{18-22}$.

Las cirugías mínimamente invasivas transanales, aunque se han llevado a cabo extremando precauciones en China, presentan un alto riesgo de generación de aerosoles y exposición directa prolongada del cirujano durante el tiempo perineal. Además, el uso de estomas (definitivas y temporales) puede generar otro foco de transmisión del virus, tanto para los cuidadores del paciente como para los trabajadores de la salud ${ }^{19,21,22}$.

De acuerdo con lo anterior, diferentes grupos quirúrgicos en el mundo han propuesto sistemas para evacuar el neumoperitoneo hacia sistemas cerrados de succión o presión negativa, con trampas de agua y filtros de alta eficiencia, que permitan un mayor grado de control de las partículas que salen de las cavidades del paciente, durante y al finalizar el procedimiento quirúrgico. Diferentes productos disponibles en el mercado podrían utilizarse también para retirar y filtrar los gases intraoperatorios como el $\mathrm{CO}_{2}$ y el humo quirúrgico, tal como lo recomienda SAGES en su última actualización el 30 de marzo de 2020, los cuales evacuan los gases de manera continua y segura durante procedimientos abiertos, laparoscópicos y endoscópicos, pero tienen un alto costo, lo que para nuestro medio puede ser una limitante 9,19.

Sin embargo, más allá de estas experiencias individuales reportadas, incluyendo el uso de insumos ya existentes en el quirófano que se adaptan para crear sistemas "hechos a mano", de bajo costo, no existen estudios comparativos que evalúen su impacto clínico, ni en términos de contagio ni en efectividad para extraer de forma segura los gases intraoperatorios ${ }^{18-22}$.

Entonces, se sugiere emplear las siguientes medidas de prevención en cirugía mínimamente invasiva (tabla 2), las cuales se han adaptado de las recomendaciones propuestas por SAGES, el RCS, la Intercollegiate General Surgery Guidance y las expuestas por Min en su publicación de Annals of Surgery para el manejo del COVID-I9 en cirugía laparoscópica ${ }^{9,13,20}$.

De acuerdo con lo anterior, al momento de escoger la vía de acceso ideal para un procedimiento quirúrgico se deben sopesar todos los factores propios de la condición del paciente, e incluir en esa balanza el riesgo de genera- 
Tabla 2. Recomendaciones para disminuir el riesgo de exposición a aerosoles en cirugía laparoscópica

\begin{tabular}{|c|c|}
\hline \multirow{5}{*}{ Manejo de la sala } & $\begin{array}{l}\text { - Idealmente los quirófanos deben organizarse con salas dedicadas a trabajar con pacientes COVID-19 } \\
\text { positivos, dado que requieren medidas extremas de precaución del contagio y un mínimo de personal } \\
\text { con alta experticia para resolver sin demora cada caso, empleando el máximo de medidas de seguridad. }\end{array}$ \\
\hline & -Asegúrese de que la comunicación entre los miembros del equipo sea óptima. \\
\hline & • Asuma que el manejo de la sala de cirugía es el mismo de una sala contaminada. \\
\hline & $\begin{array}{l}\text { - Cumpla en forma estricta los protocolos de desinfección y limpieza de quirófanos, según las re- } \\
\text { comendaciones nacionales (en tiempo, sustancias utilizadas, técnica de limpieza, desinfección y } \\
\text { esterilización). }\end{array}$ \\
\hline & • Según la disponibilidad, favorezca el uso de la sala de cirugía con presión negativa. \\
\hline \multirow{3}{*}{ Cirujano } & • Elija el equipo de protección más efectivo para prevenir el contagio a través de aerosoles. \\
\hline & $\begin{array}{l}\text { - Para la colocación y el retiro de los EPP utilice un "Sistema de amigos", en el que uno de los miem- } \\
\text { bros del equipo de trabajo lo asista al entrar y salir de la sala, verificando contra una lista de chequeo } \\
\text { que se cumplen todas las medidas de seguridad y los pasos correctos. }\end{array}$ \\
\hline & $\begin{array}{l}\text { - Adapte su técnica quirúrgica para reducir la exposición a los riesgos específicos de generación de } \\
\text { aerosoles (por ejemplo, utilizando ligaduras o clips, donde antes hubiera empleado electrocirugía) }\end{array}$ \\
\hline \multirow{8}{*}{ Cirugía } & $\begin{array}{l}\text { - Durante toda la intervención, mantenga limpios de sangre y otros fluidos corporales al máximo los } \\
\text { instrumentos quirúrgicos. }\end{array}$ \\
\hline & $\begin{array}{l}\text { - Reduzca la posición de Trendelemburg durante el máximo tiempo posible, con el fin de minimizar el } \\
\text { efecto del neumoperitoneo sobre la función pulmonar y circulatoria. }\end{array}$ \\
\hline & - Mantenga el voltaje del electrocauterio al mínimo posible. \\
\hline & $\begin{array}{l}\text { - Utilice los dispositivos de succión necesarios para reducir al máximo el humo y los aerosoles durante } \\
\text { toda la cirugía. }\end{array}$ \\
\hline & • Evite disecciones muy largas con electrobisturí para disminuir la producción de humo. \\
\hline & $\begin{array}{l}\text { - En lo posible, utilice energía bipolar o selladora de superficies ya que ésta puede disminuir la pro- } \\
\text { ducción de humo quirúrgico. }\end{array}$ \\
\hline & $\begin{array}{l}\text { - Tenga precaución en la colocación de drenajes quirúrgicos por la posibilidad de escape no controlado } \\
\text { del neumoperitoneo. }\end{array}$ \\
\hline & - Evite dispositivos de sutura para la pared abdominal que favorezcan la fuga del $\mathrm{CO}_{2}$ insuflado. \\
\hline \multirow{7}{*}{$\begin{array}{l}\text { Manejo seguro del } \\
\text { neumoperitoneo }\end{array}$} & $\begin{array}{l}\text { - Maneje en forma conservadora el neumoperitoneo: mantenga las presiones del } \mathrm{CO}_{2} \text { al mínimo posible } \\
\text { para preservar los campos de exposición quirúrgica }(10-12 \mathrm{mmHg}) \text {. }\end{array}$ \\
\hline & $\begin{array}{l}\text { - Evacúe el neumoperitoneo de manera segura, evitando las dispersión del gas mediante el uso de } \\
\text { las alternativas disponibles (filtro de alta eficiencia o trampa de agua). }\end{array}$ \\
\hline & $\begin{array}{l}\text { - Minimice el tamaño y número de las heridas quirúrgicas para disminuir el riesgo de fuga no contro- } \\
\text { lada del neumoperitoneo. }\end{array}$ \\
\hline & $\begin{array}{l}\text { - En lo posible, prefiera el uso de trocares de } 5 \mathrm{~mm} \text {, la menor cantidad y según disponibilidad, trócares } \\
\text { con balón contenedor. }\end{array}$ \\
\hline & - Retire las muestras y piezas de patología una vez que se evacue todo el $\mathrm{CO}_{2}$ y el humo. \\
\hline & - Cierre la fascia después de la extracción del $\mathrm{CO}_{2}$. \\
\hline & - Disminuya la cirugía mano asistida que puede provocar fugas significativas de $\mathrm{CO}_{2}$ insuflado y humo \\
\hline
\end{tabular}

ción de aerosoles de partículas y su potencial transmisión, pero también el impacto positivo de la cirugía de mínima invasión que permite la recuperación más temprana y en casa para muchos pacientes, $\mathrm{y}$ disminuye otras posibles fuentes de contagio intrahospitalario, que en épocas de epidemia pueden afectar tanto a paciente como a los proveedores de la salud.
Medidas que adicionalmente favorecen la utilización eficiente de las camas y del recurso humano en salud ${ }^{21,22}$.

\section{Conclusión}

Es claro que, al día de hoy, no se ha publicado información conclusiva que respalde la teoría de que los virus respiratorios se transmitan a través 
del humo quirúrgico o el neumoperitoneo. Por lo tanto, las alertas sobre los riesgos de la laparoscopia emitidas durante las primeras semanas de la pandemia deben ser analizadas con precaución y a la luz de la evidencia cambiante sobre el tema.

Tanto la cirugía abierta como la laparoscópica tienen el potencial de generar aerosoles de partículas y por lo tanto en ambos escenarios es fundamental la protección de todo el equipo de trabajo dentro del quirófano.

Existen diferentes medidas para la protección durante los PGA: los elementos de protección (EPP) se deben utilizar en todos los pacientes atendidos (incluso aquellos con baja sospecha, dada la probabilidad de que se trate de pacientes asintomáticos, pero COVID-I9 positivos). En caso de pacientes sospechosos y COVID-I9 positivos, se deben extremar las medidas de protección de todo el personal que atiende al paciente en el periodo perioperatorio.

En todos los procedimientos durante la época de pandemia, se deben buscar las estrategias más efectivas para controlar las potenciales fuentes de trasmisión y minimizar la exposición del personal en los momentos de mayor riesgo, relacionados con el manejo de la vía aérea y las cavidades del paciente ${ }^{9,15,19,21}$.

\section{Cumplimiento de normas éticas}

Consentimiento informado: Esta publicación es una revisión de la literatura, y como tal no hay necesidad de un consentimiento informado ni de aprobación del Comité de Ética Institucional.

Declaración de conflicto de intereses: Los autores no declaran ningún conflicto de interés.

Fuentes de financiación: Recursos propios de los autores.

Agradecimientos: a Juanita Ayala por la ilustración.

\section{Referencias}

I. Organización Mundial de la Salud. Prevención y control de infecciones de infecciones respiratorias agudas propensas a epidemias y pandemias en el cuidado de la salud. 20I4. Disponible en: https://www.paho.org/hq/
dmdocuments/20I4/20I4-cha-prevencion-control-atencion-sanitaria.pdf

2. Bourouiba L, Dehandschoewercker E, Bush J. Violent expiratory events: On coughing and sneezing. J Fluid Mech. 20I4;745:537-63.

3. World Health Organization (WHO). Modes of transmission of virus causing COVID-I9: implications for IPC precaution recommendations. Scientific brief. 29 March 2020. Disponible en: https://www.who.int/news-room/ commentaries/detail/modes-of-transmission-of-virus-causing-covid-I9-implications-for-ipc-precaution-recommendations

4. Del Río C, Malani PN. COVID-I9 - New insights on a rapidly changing epidemic. JAMA. 2020 February 28 [Online ahead of print]. https://doi/IO.IOOI/jama.2020.3072

5. Xiao F, Tang M, Zheng X, Liu Y, Li X, Shan H. Evidence for gastrointestinal infection of SARS-CoV-2. Gastroenterology. March 2020. https://doi/IO.IO53/j. gastro.2020.02.055

6. Brat GA, Hersey SP, Chhabra K, Gupta A, Scott J. Protecting surgical teams during the COVID-I9 outbreak: A narrative review and clinical considerations. Annals of surgery. 2020 [Online ahead of print]. Disponible en: https://umbraco.surgeons.org/media/5I64/covid-surgery_vf.pdf

7. Tang JW, Li Y, Eames I, Chan PK, Ridgway GL. Factors involved in the aerosol transmisión of infection and control of ventilation in healthcare premises. Journal of Hospital Infection. 2006;64:IOO-I4. https://doi/IO.IOI6/j. jhin.2006.05.022

8. Zemouri C, de Soet H, Crielaard W, Laheij A. A scoping review on bio-aerosols in healthcare and the dental environment. PLoS ONE. 20I7;I2: eoI780o. https:// doi/IO.I37I/journal.pone.0178007

9. SAGES. Resources for smoke \& gas evacuation during open, laparoscopic, and endoscopic procedures. 2020 March 29. Disponible en: https://www.sages.org/ resources-smoke-gas-evacuation-during-open-laparoscopic-endoscopic-procedures/

Io. Siegel JD, Rhinehart E, Jackson M, Chiarello L, and the Healthcare Infection Control Practices Advisory Committee. 2007 Guideline for isolation precautions: Preventing transmission of infectious agents in healthcare settings. Am J Infect Control. 2007;35:S65-I64. https://doi/Io.IoI6/j.ajic.2007.I0.007

II. Alp E, Bijl D, Bleichrodt RP, Hansson B, Voss A. Surgical smoke and infection control. J Hosp Infect. 2006;62:I-5.

I2. Zheng $\mathrm{MH}$, Boni L, Fingerhut A. Minimally invasive surgery and the novel coronavirus outbreak: Lessons learned in China and Italy. Annals of Surgery. 2020 Mar 26. https://doi/I0.IO97/SLA.ooooooooooo03924

I3. The Royal College of Surgeons on Edinburgh. Intercollegiate General Surgery Guidance on COVID-I9 update. Disponible en: https://www.rcsed.ac.uk/news- 
public-affairs/news/2020/march/intercollegiate-general-surgery-guidance-on-covid-I9-update consultado el 3I de marzo 2020

14. Pryor A. SAGES ans EAES recommendations regarding surgical response to COVID-I9. 2020;I-8. Disponible en: https://www.sages.org/recommendations-surgical -response-covid-r9/ [cited 2020 Mar 30].

I5. American College of Surgeons. COVID-I9 Guidelines for Triage of Emergency General Surgery Patients. Updated March 25, 2020. Disponible en: https://www.facs. org/covid-I9/clinical-guidance/elective-case/emergency-surgery

16. Asociación Española de Cirujanos. Documentos de posicionamiento y recomendaciones de la AEC en relación con la cirugía y COVID-I9. Disponible en: https://www.aecirujanos.es/Documentos-de-posicionamiento-y-recomendaciones-de-la-AEC-en-relacion-con-la-cirugia-y-COVIDi9_es_I_I52.html

I7. Royal College of Obstetricians \& Gynaecologist (RCOG) / British Society for Gynaecological Endoscopy (BSGE). Statement on gynaecological laparoscopic procedures and COVID-I9. Disponible en: https://mkobritishsociep8d9m.kinstacdn.com/wp-content/uploads/2020/03/
Joint-RCOG-BSGE-Statement-on-gynaecological-laparoscopic-procedures-and-COVID-I9.pdf

I8. American Association of Gynecologic Laparoscopists (AAGL). COVID-I9: Joint Statement on Elective Surgeries. Disponible en: www.aagl.org/news/covid-I9-jointstatement-on-elective-surgeries.

I9. Balibrea JM, Badia JM, Rubio I, Antona EM, Alvarez E, García S, et al. Manejo quirúrgico de pacientes con infección por COVID-I9. Recomendaciones de la Asociación Española de Cirujanos. Cir Esp. 2020. https:// doi/I0.IOI6/j.ciresp.2020.03.00I

20. Snyman L, Makulana T, Makin JD. A randomised trial comparing laparoscopy with laparotomy in the management of women with ruptured ectopic pregnancy. S Afr Med J. 20I7;IO7: 258-263. https://doi/I0.7196/ SAMJ.20I7.vio7i3.II447.

2I. Morris SN, Fader AN, Milad MP, Dionisi HJ. Understanding the "scope" of the problem: Why laparoscopy is considered safe during the COVID-I9 pandemic. J Minim Invasive Gynecol. 2020 Apr 2. https://doi/IO.IOI6/j. jmig.2020.04.002.

22. Brücher B, Nigri G, Tinelli A, Lapeña JF, Espin-Basany E, Macri P, et al. COVID-I9: Pandemic surgery guidance. EDP Sciences. 2020. https://doi/I0.IO5I/fopen/2020002 